\title{
LA THÉORIE AUTRICHIENNE DE LA BUREAUCRATIE DE MISES: UNE PERSPECTIVE CRITIQUE
}

\author{
LAURENT CARNIS*
}

Résumé: Compte tenu de place importante que prend désormais l'organisation étatique au sein des sociétés contemporaines, l'élaboration d'une théorie économique de la bureaucratie s'avère essentielle pour en comprendre à la fois les mécanismes de fonctionnement et les implications. Cet article revient sur la contribution de Mises, qui par ses travaux, permet d'offrir une réelle alternative aux approches traditionnelles. La première partie de cette contribution présente la théorie de Mises en soulignant ses spécificités par rapport à la conception défendue par Wéber, dont à la fois elle s'inspire et s'en écarte substantiellement. L'absence du calcul économique dans le processus d'allocation des ressources représente une dimension essentielle pour comprendre la théorie proposée. Mises démontre également que l'organisation bureaucratique s'accommode aisément des différents régimes politiques et reflète l'intervention généralisée de l'Etat.

La deuxième partie concerne les conséquences de la bureaucratie. Mises distingue quatre effets: les effets économiques, sociaux, politiques et psychologiques. Chaque catégorie fait l'objet d'une présentation détaillée.

La troisième partie soulève des points critiques du modèle de la bureaucratie de Mises. Ainsi, sa conception du bureaucrate et sa place au sein de l'organisation bureaucratique nous paraissent faire l'objet d'une élaboration insuffisante. Les mécanismes de l'expansion bureaucratique méritent également un raffinement de l'analyse, tandis que la justification avancée $d^{\prime}$ 'une bureaucratie minimale soulève la question de l'existence d'un Etat minimal.

* Chargé de recherche au groupe d'analyse du risque routier et de sa gouvernance (GARIG) à l'institut national de recherche sur les transports et leur sécurité (INRETS) et chercheur associé au laboratoire d'économie publique (LAEP) du Centre de l'Economie de la Sorbonne. L'auteur tient à remercier le Pr. Garouste et le Pr. Hüslmann, ainsi que les participants du séminaire autrichien et ceux de l'Austrian Research Seminar de Paris pour leurs commentaires. L'auteur est seul responsable des erreurs qui subsisteraient dans l'article, qui ne saurait engager l'INRETS. 
Mots clés: Bureaucratie, calcul économique, processus de marché, Etat minimal.

Abstract: The bureaucratic organisations became key actors in our contemporary societies. To develop an appropriate theory to understand how these organisations work and their implications constitute true stakes. This article deals with Mises' theory of bureaucracy, which differs radically from the traditional approaches.

The first part presents the definition of the Misesian bureaucracy, which shares some common points with Wéber's version and shows also true strong disagreements. The absence of economic calculation constitutes the main key idea to understand the Misesian theory. Mises shows also that bureaucracy reflects the governmental interventions, which can be combined with different types of political systems.

The second part deals with the consequences of bureaucracy. This part proposes a detailed analysis of the economic, social, political and psychological effects.

The third part raises some critical points for the theory. It seems that the conception of bureaucrat and its place inside the bureaucratic organisation is not sufficiently analysed. Moreover, the modelling of the bureaucratic expansion deserves a more refined research. Finally, the justifications given by Mises to justify a minimal bureaucracy emphasize the necessity to think the place and the role of the State.

Keywords: Bureaucracy, economic calculation, market process, minimal government.

JEL Classification: D73 H11 L38 P11 P21.

\section{I \\ INTRODUCTION}

L'étude de la bureaucratie est centrale pour au moins deux raisons. La première est qu'elle constitue une forme d'organisation alternative à celle de la firme ou de l'entreprise. De nombreuses études sont consacrées à cette dernière. Elle est présentée comme le lieu d'exécution d'un programme de minimisation des coûts ou de maximisation du profit (Archibald 1971). D'autres se sont intéressés aux déterminants de sa taille en intégrant les coûts de transaction ou encore les modalités de financement (Barzel, 2003), (Coase, 2000, 1960, 1937), (Alchian et Demstez, 1973). La perspective 
autrichienne insiste sur la dynamique entrepreneuriale de la firme (Sautet 2000) (Ioannides 1999). L'organisation bureaucratique a fait comparativement l'objet d'un nombre réduit d'élaborations théoriques (Greffe, 1981). Il convient donc de saisir cette réalité et de l'analyser.

Une autre raison majeure poussant à l'étude de la bureaucratie repose sur le développement contemporain de l'intervention publique et cela depuis plus de cinquante ans avec la mise en place de l'Etat- Providence (Peltzamn 1980). Or le système bureaucratique est la modalité de transmission privilégiée de l'interventionnisme public. Lorsque le gouvernement décide de produire de nouvelles lois, il doit mobiliser des structures bureaucratiques qui prennent en charge la rédaction de ces nouvelles dispositions et qui mettent en oeuvre les moyens de les faire respecter. Si le gouvernement décide de la production de nouveaux services, une structure bureaucratique sera créée ou associée. L'omniprésence de l'intervention publique nécessite de s'intéresser au phénomène bureaucratique.

Cet article présente et discute la théorie économique de la bureaucratie dans sa version autrichienne, qui est peu utilisée malgré des apports fondamentaux. En effet, le modèle théorique traditionnellement discuté en économie ou par d'autres disciplines est celui de Niskanen, c'est-à-dire une perspective se rattachant à l'approche développée par l'école des choix publics (Niskanen, $1994,1975,1968)^{1}$. La contribution majeure du courant économique autrichien a été développée dan l'ouvrage «Bureaucratie» de Mises (Mises, 2003, 1983 [1944]), même si les apports de Rothbard ne doivent pas être négligés (Rothbard 1993 [1962]). Cette théorie alternative de la bureaucratie est souvent résumée à la possibilité de l'usage du calcul économique au sein des organisations privées et publiques (Reisman, 1998 (1990), p. 304-305), voire critiquée et caricaturée (Niskanen 1994, p. 19), parfois largement ignorée et incomprise (Greffe, 1994, 1981)².

\footnotetext{
${ }^{1}$ Nous pourrions également citer les travaux de Downs (1994 [1966]), de Tullock (1965), et de Breton et Wintrobe (1982).

2 Les travaux de Mises et de Weber font l'objet d'une présentation très rapide et ne sont pas cités dans la bibliographie de l'auteur dans son ouvrage de 1981 (bien
} 
Nous proposons dans cet article de remédier dans une certaine mesure à cette incomplétude, en saisissant à la fois la particularité de l'explication autrichienne en nous limitant à sa version misessienne et $\mathrm{d}^{\prime}$ en cerner les enjeux. La dimension comparative ${ }^{3}$ avec d'autres approches est inévitable (Anderson 2004), mais nous désirons ici l'instrumenter pour mettre l'accent sur les spécificités de la démarche autrichienne. Dans une première partie, nous développons la définition et la présentation de la bureaucratie telles qu'elles sont avancées chez Mises. La deuxième partie traite des conséquences associées à l'organisation bureaucratique, tandis que dans la dernière partie de notre article nous saisissons les limites de cette approche.

II

\section{LA DÉFINITION DE LA BUREAUCRATIE}

La définition proposée par Mises partage en de nombreux points la conception wéberienne. Nous pouvons avancer l'hypothèse raisonnable d'une certaine influence de celle-ci sur les travaux de Mises. Toutefois, l'approche développée par Mises se démarque clairement des enseignements de Weber quant à l'interprétation à donner d'une telle organisation, ce qui a pour conséquence immédiate qu'il n'y a pas réduction de l'une à l'autre. Une différence essentielle réside dans l'étendue et les effets associés à l'organisation bureaucratique.

\footnotetext{
qu'apparaissant en note de bas de page), alors que le travail de Mises constitue une des premières approches économiques de la bureaucratie et que les enseignements de Weber sur le modèle bureaucratique sont incontournables en sociologie, en science politique et à certains égards en économie. L'ouvrage de 1994 ne répare pas l'outrage dans la mesure, où il le présente comme l'expression inaboutie d'une théorie, sur laquelle se développeront les efforts de formalisation de Niskanen pour en donner une expression actualisée. La thèse de l'auteur nous paraît fort discutable, qui semble s'expliquer par son ignorance des fondements à la fois du courant des choix publics et ceux de l'école autrichienne.

${ }^{3}$ Nous avions par le passé procédé à une approche comparatiste entre la théorie de la bureaucratie élaborée par Mises et le modèle de Niskanen (Carnis 2000).
} 


\section{L'influence de Weber}

Weber dresse précisément les caractéristiques de ce que recouvre une organisation bureaucratique et donc par ce qu'il entend par bureaucrate (Weber, 1952, p. 196-198). Le principe essentiel est l'articulation du fonctionnement de l'organisation autour de règles, de lois ou de réglementations publiques. Les attributions du bureaucrate sont enchâssées dans des procédures et sont contrôlées par l'autorité hiérarchique, laquelle prend la forme d'une pyramide dont le contrôle s'exerce du haut vers le bas. Le bureaucrate ou le fonctionnaire est un élément essentiel de la machine bureaucratique (Ibid. p. 198-204). La profession de fonctionnaire relève d'une véritable vocation et nécessite des compétences particulières qui font l'objet d'un contrôle. Le bureaucrate est compétent, bénéficie d'une estime sociale associée à l'occupation du poste, lequel est rémunéré non pas en fonction d'un niveau de production, mais qui dépend du statut et de son ancienneté. La fonction s'accompagne d'un certain nombre d'avantages comme le bénéfice d'un emploi à vie et d'une pension de retraite.

Pour Weber, la bureaucratie est le produit de l'histoire, l'expression de la forme ultime de gestion des affaires publiques. La bureaucratie publique est l'expression d'une rationalité légale, où la loi permet l'égalité des citoyens. L'exécution des tâches administratives est dépersonnalisée, permettant d'éviter les situations de corruption et de favoritisme. La bureaucratie wéberienne prend la forme d'une mécanique froide, impersonnelle dont la course ne peut être facilement déviée.

«... the more the bureaucracy is "dehumanised", the more completely it succeeds in eliminating from official business love, hatred, and all purely personal, irrational, and emotional elements which escape calculation. This the specific nature of bureaucracy and it is appraised as its special virtue.» (Ibid. p 216)

Pour conclure sur cette présentation rapide de la bureaucratie chez Weber, il est essentiel de souligner que le phénomène bureaucratique ne se limite pas à la gestion publique. Définissant 
la bureaucratie par certaines caractéristiques organisationnelles, il en identifie des éléments au sein des grandes entreprises. Pour distinguer les deux formes, il parle $\mathrm{d}^{\prime}$ "autorité bureaucratique» pour désigner en fait l'administration et de «management bureaucratique» pour les entreprises (Weber 1952, p. 196).

Mises reprend pour partie les caractéristiques développées par Weber afin de comprendre la bureaucratie. Pour Mises, la bureaucratie se présente comme un mode d'administration des ressources qui s'appuie largement sur une codification des tâches à exécuter et des règles de comportement. L'importance de la règle prédomine.

«L'organisation bureaucratique est celle ou les responsables sont tenus de se conformer aux statuts et règlements détaillés établis par l'autorité d'un organisme supérieur. Le rôle du bureaucrate est d'exécuter les prescriptions des statuts, et règlements, qui restreignent singulièrement pour lui le pouvoir $\mathrm{d}^{\prime}$ agir dans le sens qu'il estime le meilleur.» (Mises, 2003 [1944] p. 53)

Les règlements constituent ainsi la courroie de transmission des décisions de l'autorité supérieure ${ }^{4}$ par le biais de la chaîne hiérarchique tout en maintenant une action dépersonnalisée sans égard au caractère juste ou injuste de l'intervention. Le bon bureaucrate est celui qui applique les règlements.

«Leur préoccupation principale est d'obéir aux règlements et ordonnances qu'ils soient raisonnables ou contraires aux buts poursuivis. La première qualité d'un administrateur est l'obéissance aux codes et aux décrets.» (Ibid., p. 47)

Sans doute marqué par le contexte historique dans lequel Weber écrit, le bureaucrate est également présenté comme un administrateur qui occupe un poste dont la particularité s'explique par le fait que le produit de son travail ne peut pas être apprécié

\footnotetext{
${ }^{4}$ Mises conçoit ainsi les règlements et les lois mis en œuvre par la bureaucratie comme «des instruments grâce auxquels s'exécute la volonté de l'autorité supérieure». (p. 51).
} 
en termes monétaires (Ibid. p. 60). La fonction est donc rémunérée selon des considérations statutaires et non selon des critères productifs. Son recrutement est réalisé par une autorité supérieure. Là où Weber associe une certaine estime sociale à l'occupation $\mathrm{d}^{\prime}$ un tel poste, auquel sont associés une rétribution avantageuse et d'autres bénéfices, qui peuvent être interprétés comme la contrepartie de la compétence et du dévouement dont fait preuve le bureaucrate. Il n'y a rien de tel pour Mises. Pour ce dernier, le terme de bureaucrate est devenu péjoratif. Il affirme d'ailleurs:

«Nul ne doute que la bureaucratie ne soit on ne peut plus néfaste, ni qu'elle doive disparaître d'un monde parfait.» (Ibid., p. 5)

Plus en avant dans l'ouvrage (Chap. V), Mises développe l'intérêt recherché par les postulants à la carrière de fonctionnaire. Les avantages divers, un salaire plus élevé, une retraite assurée et confortable, des conditions de travail moins pénibles et une sécurité de l'emploi présentent des attraits évidents pour certaines personnes. Bien que l'analyse de Mises s'accorde sur les caractéristiques d'une bureaucratie que Weber a préalablement cernées, l'interprétation est toute différente.

Un autre point commun aux deux analyses concerne l'identification de formes bureaucratiques au sein des grandes entreprises. Cependant, l'interprétation qui en est donnée s'avère radicalement différente. Pour Weber, il s'agit de l'identification de mécanismes organisationnels (ligne hiérarchique, lien de subordination...) communs, des mécanismes administratifs similaires à ceux existant dans l'appareil d'Etat et qui témoignent d'une certaine efficacité. Pour Mises, ils ne représentent que les conséquences de l'intrusion de l'Etat dans le fonctionnement des affaires privées. Sans l'intervention de l'Etat dans l'économie et le monde des affaires, ces fonctions n'auraient aucune raison d'être au sein des entreprises privées, car fondées sur aucune justification de profit.

«L'objet du présent ouvrage est de tenter de démontrer que, pour l'entreprise animée par la recherche du profit, le danger de la bureaucratie n'existe pas à la condition que l'Etat laisse à ses 
dirigeants les mains libres. L'entreprise privée n'évolue pas nécessairement vers une structure bureaucratique rigide. La tendance dénoncée résulte de l'immixtion de l'Etat dans le secteur privé. Elle est le fruit de la politique qui tend à enlever au mobile du profit le rôle qu'il joue dans l'organisation économique de la société. » (Mises 2003 (1944) p. 17).

\section{La définition de la bureaucratie chez Mises}

Même si Mises ne donne pas de définition précise de la bureaucratie, il souligne qu'il s'agit d'un mode d'allocation des ressources, " une méthode de gestion applicable en différents secteurs de l'activité humaine » (Ibid. p. 50). La bureaucratie est " un principe de technique et d'organisation administrative ". Mises en donne également une définition négative, à savoir ce qu'elle n'est pas à défaut de préciser ce qu'elle est.

«L'organisation bureaucratique est la méthode appliquée à la conduite des affaires administratives dont le résultat ne s'apprécie pas en monnaie sur le marché.»

«L'organisation bureaucratique est la conduite des affaires qui échappent au contrôle du calcul économique.» (Ibid. p. 54)

En conséquence, la gestion bureaucratique se définirait comme l'allocation de ressources qui n'obéirait pas au critère du profit. En fait, une définition plus précise de la bureaucratie chez Mises consiste en la procédure d'allocation ${ }^{5}$ de ressources non légitimement possédées par l'administrateur, qui procède par l'intermédiaire d'un système de règles régit par des relations de commandement ${ }^{6}$. L'allocation n'obéit pas à un principe de

5 Le terme allocation ici ne désigne pas seulement l'attribution directe de ressources à certains usages, mais également l'obligation ou l'influence directe de réglementations à la direction de ressources à des usages particuliers.

6 Les grandes entreprises constituent-elles des bureaucraties ? En effet, les dirigeants sont généralement des managers qui ne sont pas propriétaires de l'entreprise, au sein desquelles des règles existent ainsi que des relations hiérarchiques. En fait, l'embauche du manager repose sur une relation contractuelle qui lui impose 
valorisation par le marché et est indifférente à la propriété privée ${ }^{7}$.

«One must first be clear as to what is really to be understood by bureaucracy and the bureaucratic conduct of business, and just how these are distinguished from commercial enterprise and the commercial conduct of business. The opposition between the commercial and the bureaucratic mentality is the counterpart in the intellectual realm of the opposition between capitalism - private ownership of the means of production - and socialism - communal ownership of the means of production.» (Mises, 1996 (1985) [1927]).

La définition proposée met en évidence trois caractéristiques essentielles de la bureaucratie, à savoir la violation de droits de propriété, des dépendances hiérarchiques et une allocation systématique des ressources par des règlements. En cela, une gestion bureaucratique ne nécessite pas nécessairement la production de biens ou de services par une entreprise publique. Une entreprise privée peut être obligée par une loi à dépenser une partie de ses ressources à la protection de l'environnement. Dans ce cas, il y a immixtion de l'Etat dans les affaires privées de l'entreprise, qui de fait se voit expropriée d'une partie de sa propriété légitime. Ces critères permettent à Mises de distinguer ainsi une gestion par le système du profit et une gestion bureaucratique, dont le Tableau 1 précise les distinctions fondamentales.

de gérer au mieux commercialement l'entreprise. Certes des règles et une hiérarchie existent, mais elles ne sont pas les éléments essentiels de leur fonctionnement. L'organisation de l'entreprise doit prendre en compte une logique commerciale, comptabiliser les coûts de production et les produits des ventes. Si l'entreprise abandonne partiellement cet objectif commercial, elle intègre alors des éléments constitutifs d'une bureaucratie au sens de Mises.

7 Même si Mises ne développe pas une théorie des droits de propriété, le droit de propriété est au cœur de son analyse et de son argument sur le calcul économique. Les droits de propriété sur les facteurs de production s'avèrent à cet égard fondamentaux. "The liberals maintain that the only workable system of human cooperation in a society based on the division of labor is private ownership of the means of production.» (Mises 1996, p. 18). Notre analyse diffère substantiellement de celle du Pr. Hülsmann sur ce point pour qualifier l'organisation bureaucratique. 
TABLEAU 1

LES CARACTÉRISTIQUES D'UNE GESTION

BUREAUCRATIQUE ET COMMERCIALE

\begin{tabular}{|c|c|c|}
\hline & Gestion bureacratique & Gestion marchande \\
\hline Régime de propriété & Violation, imposition & $\begin{array}{l}\text { Relation contractuelle } \\
\text { et respect de la propriété } \\
\text { privée }\end{array}$ \\
\hline Mode d'allocation & Règles & Système de prix \\
\hline Mode d'organisation & $\begin{array}{l}\text { Relation hiérarchique } \\
\text { et de commandement }\end{array}$ & Relation commerciale \\
\hline Création de valeur & $?$ & $\begin{array}{c}\text { Indicateur de profit } \\
\text { et expression monétaire }\end{array}$ \\
\hline
\end{tabular}

La gestion commerciale s'appuie sur des droits de propriété, un système de prix qui permet l'allocation des ressources aux usages les plus valorisés. Il s'adosse à des relations commerciales (concernant le contrat de travail, les ventes...), dont la valorisation peut prendre une expression monétaire. La création de valeur associée à la gestion bureaucratique est plus problématique à démontrer dans la mesure où celle-ci ne peut prendre d'expression monétaire.

«Nous ne disons pas, remarquons-le, qu'une conduite heureuse des affaires publiques n'a pas de valeur; nous disons que le marché n'en indique pas le prix, que la valeur n'en peut être réalisée dans une transaction commerciale et n'est par conséquent pas susceptible d'expression en termes monétaires.» (Mises 2003 [2004] p. 53)

La gestion bureaucratique implique des relations de coercition, la violence, le non-respect des droits de propriété, des relations de hiérarchie et de commandement. 


\section{Les différences de typologie entre les approches de Mises et de Weber}

La terminologie de gestion bureaucratique et commerciale proposée par Mises peut prêter à confusion avec la typologie avancée par Weber. Pour Weber, la gestion bureaucratique signifie qu'une entreprise privée est organisée autour de principes bureaucratiques tels qu'il les a définis. Lorsque ces principes sont mis en œuvre dans les organisations étatiques, Weber qualifie cette situation d'autorité bureaucratique.

Pour Mises, la gestion bureaucratique peut caractériser certaines entreprises privées et peut concerner également les administrations publiques. En cela, ce que Mises exprime par gestion bureaucratique peut inclure à la fois les concepts d'autorité bureaucratique et de gestion bureaucratique de Weber et recouvre les modalités $\mathrm{d}$ 'allocation des ressources par des règles. Ainsi, la gestion bureaucratique de Mises renvoie à la fois à la gestion bureaucratique et l'autorité bureaucratique de Weber. De même, la notion wéberienne de gestion bureaucratique peut recouvrir les concepts de gestion bureaucratique et de gestion commerciale développés par Mises, lorsqu' elle se limite à une procédure administrative.

En somme, la vision wéberienne participe d'une approche organisationnelle de la bureaucratie alors que l'analyse de Mises consiste en une approche fonctionnelle (rôle des prix et des droits de propriété). Il nous semble cependant que la définition wéberienne établit une assimilation abusive de la notion de règles au sein des organisations privée et publique. En effet, la règle au sein de l'organisation publique est souvent synonyme de réglementations, de règlements, de loi qui permettent de définir des procédures au caractère impératif et juridique. La règle au sein d'une organisation privée se réfère le plus souvent à un processus, voire des procédés, c'est-à-dire un agencement d'opérations plus ou moins complexes qui peuvent évoluer en conséquence si l'atteinte de l'objectif est plus aisée. Son évolution est d'autant plus facile que l'exécutant puisse faire des propositions pouvant être retenues par la direction (système de management de Toyota par exemple ). Ceci n'est pas le cas dans une structure bureaucratique, dans la mesure où l'obéissance de l'exécutant est primordiale. 
Une éventuelle discussion de la règle est alors interprétée comme sa remise en cause. Cette distinction souligne que quand bien même l'approche de Mises serait influencée par les travaux de Weber, elle ne se réduit pas à celle-ci ${ }^{8}$.

\section{Le phénomène bureaucratique: cause ou symptôme d'un mal plus profond, l'interventionnisme}

Pour Mises, la bureaucratie ne constitue pas le véritable problème auquel la société doit faire face. En effet, elle «n'est qu'un corollaire et un symptôme de transformations plus profondes» qui est à l'œuvre, à savoir le glissement vers une économie socialiste avec un Etat omnipotent et totalitaire ${ }^{9}$.

«Mais la bureaucratie n'y est pour rien. C'est le fait du nouveau système de gouvernement qui restreint la liberté de l'individu dans la direction de ses propres affaires et assigne à l'Etat des tâches toujours plus lourdes. Les responsable n'est pas le bureaucrate, mais le système politique.»

«La bureaucratie joue un rôle essentiel dans l'exécution de ses plans. Mais on a tort d'imputer aux bureaucrates pris individuellement les torts $\mathrm{d}^{\prime}$ un système.» «C'est le système qui est mauvais, et non les sous-ordres qu'il utilise.» (Mises 2003 [1944] p. 23).

Ainsi, la bureaucratie n'est que l'expression de l'interventionnisme public, dont elle est une modalité d'exécution particulière. Les organisations bureaucratiques sont d'autant plus prépondérantes dans l'économie que l'interventionnisme public est répandu. L'existence de structures bureaucratiques signifie également l'atteinte au droit des personnes, une restriction de leur liberté, mais également un usage d'autant limité du mécanisme des profits et des pertes, et une mauvaise allocation des ressources au sein de l'économie. Les effets de la gestion bureaucratique sont d'autant plus néfastes que

\footnotetext{
8 Pour un étude comparative des théories de Mises et de Weber sur leur analyse de la bureaucratie, voir Anderson (2004).

${ }^{9}$ Hayek avait établi un constat similaire au même moment (Hayek 1993 [1944]).
} 
l'interventionnisme public est répandu. Ainsi au-delà des effets liés à la nature de la relation bureaucratique, il existe un impact lié au degré de bureaucratisation d'une société. Une dynamique propre à la sphère étatique est alors en marche. Une généralisation de la production bureaucratique implique une atteinte généralisée à la souveraineté des consommateurs et des droits de propriété, une socialisation progressive de l'appareil de production pour laquelle la signification du mécanisme des prix est d'autant plus réduite que le marché libre est limité à une portion congrue du système économique. Dans cet environnement particulier, le comportement des bureaucrates s'adapte en conséquence au système et aux mécanismes d'incitations qui ne relaient pas les préférences des consommateurs. Ils constituent alors les rouages d'une énorme machine dont les conséquences seront analysées dans la section suivante (Figure 1).

Mises justifie l'existence d'une bureaucratie, qui serait nécessaire pour assurer la coopération sociale et éviter une situation anarchique. En somme, l'intervention publique doit se limiter à assurer les tâches de police et de justice, et de défense (Mises 1996 (1985) [1927] p. 36-38 et p. 84). Il s'agit de préserver la propriété, la paix et la liberté et d'éviter que la société sombre dans l'anarchie.

FIgURE 1

LES CONDITIONS D'EXISTENCE

DE LA BUREAUCRATIE

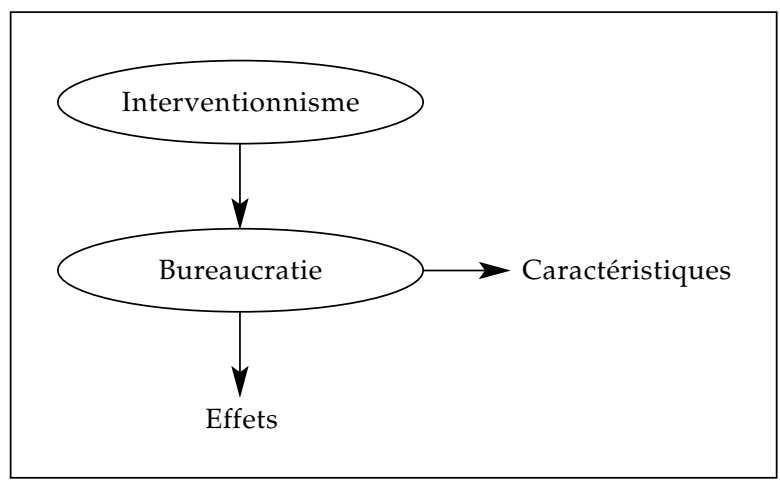


«Et comme la collaboration sociale, pour être efficace, nécessite un gouvernement, une certaine dose de bureaucratie est indispensable. Ce dont nous souffrons, ce n'est pas tant de la bureaucratie ellemême que de son intrusion dans tous les domaines de la vie et de l'activité humaines.» (Mises, 2003 [1944] p. 23)

Selon Mises, une bureaucratie réduite fonctionne à peu près convenablement dans la mesure où les décisions prises peuvent s'adosser à l'information véhiculée et générée par le fonctionnement du marché. Sans cette information relative au système des prix relatifs, " une économie sans calcul en termes de monnaie ne peut exister que dans des conditions très simples » (Mises, 1939, p. 106 et s.). En somme, Mises pose les limites de l'extension de la bureaucratie à la fois par un argument positif et négatif. La bureaucratie trouve ses limites dans la protection des biens et les personnes et ne peut fonctionner de manière économiquement rationnelle qu'en s'appuyant sur l'information générée par le fonctionnement de l'économie de marché. Au-delà et à mesure de son extension, l'économie se dirige alors vers un chaos généralisé (Mises 2003 [1944], p. 65). L'économie contrôlée ne dispose pas des moyens de connaître si les plans de production sont profitables ou non. Par ailleurs, par ce que la gestion bureaucratique implique une violation du droit de propriété ${ }^{10}$ et une redistribution autoritaire de revenu (Mises, 2002 [1928], p. 372 et s.), le franchissement des limites d'une bureaucratisation minimale signifie un changement de nature: elle ne vise plus à une protection et à la production d'un bien nécessaire. Elle crée désormais de l'insécurité économique par son impossibilité à corriger les erreurs et de l'insécurité politique en violant les droits de propriété.

10 Mises n'utilise pas ces mots. Néanmoins, il utilise régulièrement le qualificatif de «souveraineté du consommateur» pour désigner le pouvoir d'influence du consommateur sur le producteur afin que ce dernier produise le bien désiré. Selon Mises, une conséquence de l'interventionnisme réside dans la disparition de cette souveraineté. Nous pouvons traduire sans trahir la pensée de l'auteur, qu'il s'agit bien d'une violation de ses droits à l'allocation de ces ressources. Ceci est encore plus flagrant, lorsque la dépense publique se fait au détriment de l'individu. «Suffice it to say here that the "general economic" objectives of public enterprises amount to the subsidization of one group of citizens at the expense of the rest» (Mises, 2002 [1928], p. 377). 


\section{Les différentes combinaisons politiques et économiques de la bureaucratie}

Le système bureaucratique est un mécanisme de transmission particulier de l'interventionnisme public, qui ne prend pas en compte la nature des objectifs à atteindre. Il peut donc être associé à la fois à divers systèmes politiques et s'étendre à différentes formes d'entreprise.

Il peut soutenir un système totalitaire ou un Etat constitutionnel. La bureaucratie n'est qu'un système d'exécution qui peut s'avérer redoutable, d'autant plus lorsqu'il est au service de l'injustice.

«L'Etat est la seule institution qualifiée pour user de la contrainte et infliger des peines aux individus. Ce pouvoir effrayant ne peut être abandonné à l'arbitraire de quelques hommes, si compétents et si intelligents qu'ils s'estiment eux-mêmes. Il est nécessaire de réduire son étendue.» (Ibid. p. 84)

Mises insiste sur cette distinction pour éviter la confusion entre bureaucratie et système totalitaire.

«Le totalitarisme est bien autre chose que la simple bureaucratie. $C^{\prime}$ est dans la soumission totale de l'individu, dans le travail et dans le loisir, aux ordres des dirigeants et des fonctionnaires. Il réduit l'homme à n'être qu'un rouage dans un mécanisme de contrainte et de coercition qui embrasse tous les aspects de la vie individuelle. Il oblige l'individu à renoncer à toute activité que l'Etat n'approuve pas. Il transforme la société en une armée du travail admirablement disciplinée, disent les défenseurs du socialisme, en un bagne répliquent ses adversaires». (Mises, 2003 [1944], p. 23)

En conséquence, le caractère bureaucratique n'est pas le critère discriminant pour établir le caractère despotique d'un système politique. Le critère essentiel de l'analyse de Mises est la souveraineté du peuple. La loi est ce qui permet alors de protéger l'individu de l'arbitraire de l'administration et du bureaucrate. L'administration se voit donc contrainte dans l'utilisation et le montant des dépenses. Le parlement se doit de 
jouer le rôle de contrôleur général des dépenses et veiller à ce qu'il ne se produise pas de dérives budgétaires.

«Le trait caractéristique (de l'Etat constitutionnel) qui le distingue du despotisme est que ce n'est pas l'administration, mais les représentants du peuple régulièrement élus qui ont à déterminer ce qui sert le mieux le bien public. C'est le seul système qui réalise la souveraineté du peuple et sauvegarde son droit à se gouverner lui-même.» (Ibid. p. 49)

Mises donne donc un tour plus politique à l'analyse de la bureaucratie et ne se limite pas à la seule dimension économique. Il y a cependant une caractéristique commune à ces deux dimensions. Il s'agit de la souveraineté de l'individu, en tant qu'électeur lorsqu'il élit ses représentants au parlement, en tant que consommateur lorsqu'il choisit les biens et services qu'ils désirent consommer. La souveraineté de l'individu peut être considérée comme la capacité de contrôle de celui-ci sur ses ressources, qui peut prendre une forme directe par son comportement d'achat et sa capacité à négocier pour des biens privés et une forme indirecte en élisant des représentants contrôlant la dépense budgétaire concernant la consommation de biens publics.

La bureaucratie peut être déclinée également selon différents types d'organisation. Ainsi, il distingue une bureaucratie au sein de l'administration, qui regroupe les agences gouvernementales, les ministères et les collectivités locales et territoriales, dont les prestations ne peuvent être appréciées en termes monétaires. Les entreprises publiques constituent également une forme de bureaucratie, dont le produit est rarement supérieur à la dépense. L'objectif de ces entreprises consiste à procéder à un transfert de revenu entre les individus.

«Quels que puissent être les autres buts poursuivis, une telle politique revient toujours en définitive à subventionner les uns aux frais des autres.» (Ibid. p. 68)

En effet, leur objectif n'est pas de rechercher un profit, mais de produire le plus possible ou d'offrir le produit ou le service 
à un prix relativement bas. La réalisation d'une perte ne s'en trouve pas sanctionnée. Au contraire, elle peut refléter une pratique tout à fait satisfaisante de la part des autorités, à savoir «la stricte obéissance à un ensemble de règles» et «une soumission à cette réglementation».

La bureaucratie concerne également les entreprises privées. Lorsque l'entreprise est détournée de son objectif commercial et donc d'entreprendre une activité profitable, l'entreprise compose avec les influences de la bureaucratie (Mises, 2002 [1928], p. 378 et 379), (Mises 1996 (1985) [1927], p. 103). Mises précise ainsi que l'entreprise privée peut être entravée dans son fonctionnement de différentes manières: par une limitation de son profit, par un contrôle des prix, par le contrôle du taux de marge en influençant les coûts de production ou en accroissant le taux d'imposition payé par les entreprises (Mises 2003 (1944) p. 75). Mises souligne que cette politique a pour conséquence de limiter le rythme d'accumulation du capital du fait de distorsions fiscales et de réduire les incitations des entrepreneurs à prendre des risques et à se lancer dans des activités productives.

L'influence bureaucratique conduit également les entreprises privées à modifier la gestion de leur personnel. Il s'agit dès lors de respecter des règles définies par l'Etat et d'infléchir les efforts en faveur de l'atteinte des objectifs commerciaux. L'immixtion de la bureaucratie dans la gestion du personnel prend une forme plus pernicieuse, lorsqu'elle oblige l'entreprise à rechercher des appuis politiques et syndicaux. Les conseils d'administration doivent faire une place à certains amis politiques, les entreprises doivent procéder à des versements en faveur de partis politiques et de syndicats pour s'assurer des protections nécessaires ou pour protéger le marché sur lesquels elles interviennent (Ibid. p. 78-80).

Le contrôle bureaucratique peut conduire à une subordination totale de l'entreprise privée à l'administration. Celle-ci dispose en effet d'un arsenal législatif qui peut conduire l'entreprise à de graves difficultés, voire à sa fermeture ou encore à sa faillite, mais également à l'expropriation de son légitime propriétaire. Il s'agit alors pour le dirigeant de saisir au mieux les opportunités, d'user de diplomatie et de corruption parfois, pour éviter la perte de son affaire. 
III

\section{LES CONSÉQUENCES DE LA BUREAUCRATIE}

Mises consacre une large part de son ouvrage aux conséquences néfastes de l'organisation bureaucratique, concernant les dimensions économiques, politiques, sociales et psychologiques.

\section{Les conséquences économiques}

Pour comprendre les conséquences économiques de la bureaucratie, Mises établit une comparaison systématique entre le management privé et l'organisation bureaucratique pour en souligner les logiques divergentes.

Mises rappelle que l'économie capitaliste ou de marché est fondée sur la propriété privée des moyens de production. Le marché repose sur un système de coopération sociale et de division du travail (Mises 2003 (1944) p. 25). Au sein de ce système, l'entrepreneur joue un rôle essentiel pour satisfaire les besoins des consommateurs. Car ce sont les consommateurs qui orientent les efforts de la production dans les différents secteurs par le mécanisme que constitue le système de prix relatifs. Le but de l'entrepreneur est la réalisation d'un profit, à savoir assurer un niveau de produit supérieur à celui des débours (Ibid. p. 25). La contrainte de la recherche du profit oblige l'entrepreneur à satisfaire le consommateur, qui agit en véritable souverain dans la mesure où il dispose du pouvoir d'allouer son pouvoir d'achat là où bon lui semble. Certes l'entrepreneur assure les décisions techniques de la production, mais ce sont les consommateurs qui orientent la production tant sur le plan quantitatif que qualitatif.

«Capitalistes, entrepreneurs, et exploitants sont des instruments dans la conduite des affaires économiques. Ils sont à la barre et dirigent le navire, mais ils n'ont pas la liberté de lui fixer un itinéraire. Le commandement en chef ne leur appartient pas, ils ne sont que des hommes à la barre tenus d'obéir sans réserve aux ordres du capitaine. Le capitaine, c'est le consommateur.» (Ibid.) 
Le système récompense ainsi l'entrepreneur qui fait des choix judicieux par le profit ou le sanctionne lorsqu'il a pris des décisions malheureuses par une perte. Il permet d'orienter les ressources productives vers les secteurs qui satisfont les besoins les plus urgents.

«Nul entrepreneur ne peut investir son capital dans une affaire qui n'offre pas de perspectives de profit. C'est précisément cela qui fait la souveraineté du consommateur et contraint le chef d'entreprise à produire l'article que les consommateurs réclament le plus instamment.» (Ibid. p. 30)

Les traits essentiels de l'économie de marché sont donc la capacité du consommateur à pouvoir orienter les ressources productives à la satisfaction des besoins jugés les plus urgents. Cette souveraineté des consommateurs prend une forme directe par l'intermédiaire de la dépense. Le système du profit traduit les préférences des consommateurs et oriente le producteur à établir des choix adéquats. Une tout autre caractéristique essentielle de l'économie de marché reste toutefois la propriété privée, pour les consommateurs avec leur pouvoir d'achat et pour les producteurs qui engagent leurs ressources et qui peuvent s'approprier le surplus.

La supériorité du système capitaliste sur tout autre système réside dans sa capacité à rendre possible le calcul économique. Le système de prix permet à la fois de s'assurer de la viabilité des plans de production présents que de la rentabilité des projets. Le mécanisme du calcul économique concerne l'ensemble des entreprises, quelle que soit leur taille. Le calcul économique permet de cerner les plans de production satisfaisants les besoins les plus urgents parmi l'ensemble des besoins exprimés sur le marché et qui peuvent être contradictoires. Il permet de sélectionner la technique productive adéquate, mais également de déterminer les quantités devant être produites et leurs qualités.

«La supériorité du système capitaliste réside en ce qu'il est le seul système de coopération sociale et de division du travail qui permette le calcul de l'imputation, qu'il s'agisse d'élaborer de nouveaux 
programmes de production ou d'évaluer les services rendus par des usines, les exploitations et les ateliers qui fonctionnent déjà.» (Mises, (2003) 1944, p. 27)

Contrairement au système de marché, la gestion bureaucratique se révèle incapable d'évaluer les plans de production. Plus précisément, le calcul économique n'est possible que sur une dimension réduite du développement de la bureaucratie, c'està-dire lorsqu'elle se réduit aux dimensions de défense, de police et de justice (Mises, 1996 (1985) [1927]). Dans cette situation, l'organisation bureaucratique bénéficie de l'information générée par le marché. A mesure du développement de l'immixtion bureaucratique, l'information générée par le marché se dégrade et devient de ce fait peu pertinente. Le calcul économique ${ }^{11} \mathrm{~s}^{\prime}$ avère dès lors impossible et les plans de production idoines ne peuvent être choisis. Le système de perte et de profit ne peut être utilisé, là où il était primordial pour l'allocation des ressources. En conséquence, dans une économie socialiste où l'organisation bureaucratique est généralisée, le calcul économique est tout bonnement impossible.

«En régime socialiste, on ne peut discerner ni les profits, ni les pertes. Là où le calcul fait défaut, il n'y a aucun moyen de savoir si les programmes conçus ou exécutés étaient ceux qui convenaient le mieux à la satisfaction des besoins les plus pressants ; le succès ou l'échec restent dans l'ombre.» (Mises, 2003 (1944), p. 34)

Non seulement, le marché sélectionne les plans de production adéquats, mais corrige aussi les erreurs éventuelles commises par les entrepreneurs. En conséquence, la gestion bureaucratique s'avère incapable de déterminer la rentabilité des plans de production, car elle ne dispose pas des mécanismes de correction, ce qui laisse place à des phases de cycle économique

11 Un calcul économique public peut être mis en œuvre, mais il ne reflète aucunement les préférences des consommateurs et s'établit sur des données erronées. En cela, il ne peut et ne doit pas être confondu avec le calcul économique d'un système de libre marché. 
chaotique, de développement étatique suivi d'ajustements brutaux (Hülsmann, 1998). Si l'entrepreneur satisfait les consommateurs, il dispose alors d'un profit. Il s'ensuit qu'un état moins satisfaisant est remplacé par un autre qui l'est plus. Si la mauvaise appréciation de la situation par l'entrepreneur se traduit par une perte, alors on peut considérer que ce dernier a subventionné une partie de sa clientèle. Dans ce cas, les consommateurs servis sont satisfaits. Seul l'entrepreneur subit une perte qui résulte de mauvais choix passés. Le fonctionnement du mécanisme de prix et la possibilité du calcul économique suggèrent que seul un système d'économie de marché puisse créer de la richesse.

Il en est tout autrement dans le cadre d'une gestion bureaucratique. En effet, les ressources sont allouées de manière autoritaire. Il n'existe aucun moyen de savoir si les productions sont adéquates et réalisées avec la technique de production appropriée. Même si la production n'est pas sans valeur, sa valorisation ne peut faire l'objet d'une monétarisation. En conséquence, il peut être avancé que celle-ci ne peut que générer une perte. En effet, d'un point de vue logique, la décision de bureaucrate(s) ne peut pas refléter celle de l'ensemble des consommateurs. En somme, il pourrait déterminer une quantité approximative des besoins sans disposer toutefois des caractéristiques des biens désirés, ni même connaître si l'emploi des ressources utilisées est satisfaisant. La gestion bureaucratique implique en conséquence une situation généralisée de subvention des besoins des uns par les moyens des autres. Certains biens sont produits en quantités trop importantes, $\mathrm{d}$ 'autres avec une utilisation trop intensive de facteurs de production ou encore réalisés dans des délais trop longs. Les pertes sont donc généralisées et systématiques. Un point de tension subsiste cependant dans la présentation de Mises quant aux effets de la bureaucratie. En effet, la mauvaise appréciation de la situation par l'entrepreneur peut engendrer une perte au même titre qu'une gestion bureaucratique. $N^{\prime}$ existe-t-il pas une proximité entre les deux modes d'allocation qu'il ne le semble à première vue? En fait, il existe une véritable différence entre ces deux situations. Premièrement, la perte supportée par l'entrepreneur est le fait de sa propre propriété. Il supporte sur ses fonds propres les 
conséquences de son erreur. Le consommateur bénéficie d'une subvention dans la mesure où il bénéficie d'un bien dont le prix de revient est inférieur à son coût de production. Dans une organisation bureaucratique, le bureaucrate ne supporte pas la perte, mais la reporte sur les contribuables. Deuxièmement, les pertes subies par les entrepreneurs sont d'une importance relativement réduite par rapport à celles qui peuvent être générées par un système économique complètement socialisé, par ce qu'elles sont détectées et corrigées rapidement. Troisièmement, la perte enregistrée par l'entrepreneur survient ex post, c'est-àdire que le plan de production initié n'a pas été jugé satisfaisant par les consommateurs. En ce qui concerne la bureaucratie, il s'agit d'une perte ex ante dans la mesure où l'allocation des ressources est réalisée en l'absence d'orientations de la part du consommateur d'une part, et son financement est assuré à partir de ressources (fiscales) dont l'orientation initiale a été modifiée, d'autre part.

La supériorité de l'économie de marché sur l'organisation bureaucratique repose sur la possibilité du calcul économique, à savoir la possibilité de comparer en termes monétaires les différents usages des ressources. Ce système n'est pas uniquement à l'œuvre sur le marché, mais aussi au sein des entreprises. Il irrigue chaque unité productive par l'intermédiaire du principe de comptabilité, qui enregistre les produits et les pertes. Il incite donc chaque directeur d'unité à dégager des profits afin d'éviter de perdre la direction de celle-ci. L'argument avancé par Mises est intéressant, puisqu'il suggère que l'efficacité du calcul économique ne dépende pas de la taille de l'entreprise. Il irrigue l'ensemble des structures et au sein de celles-ci. Ainsi, le système de comptabilité permet d'éviter la présence de structures bureaucratiques au sein des grandes entreprises et assure une gestion décentralisée.

Mises distingue différentes immixtions de la bureaucratie. La première forme renvoie aux administrations publiques composées d'agents qui définissent et appliquent des règles. La seconde est constituée des entreprises publiques où l'obéissance à la règle s'avère plus importante que la recherche du profit. Les entreprises privées font également l'objet d'une procédure 
de bureaucratisation, qui conduit à substituer l'obéissance à certaines réglementations à la recherche du profit.

Le contrôle étatique se traduit également par des conséquences sur la politique de recrutement des entreprises privées. Il ne s'agit plus de prendre en considération le produit actualisé du travail et de le rapprocher de son coût pour décider de l'embauche éventuelle. Dans un système bureaucratisé, l'entreprise recherche en premier lieu l'appui des syndicats et celui des partis politiques auxquels elle verse des contributions et les associe à ses conseils d'administration. La relation de dépendance à l'égard du politique se transforme progressivement en un lien de subordination. Il $\mathrm{s}^{\prime}$ agit ainsi de travailler à l'élaboration d'une réglementation ou de rechercher la protection publique sur un secteur d'activité au détriment des concurrents et des consommateurs.

Ces interventions étatiques dans le bon fonctionnement de l'entreprise privée la conduisent vers un processus de bureaucratisation. Alors que l'entreprise doit être "pilotée» directement par le fonctionnement du marché, celle-ci consacre désormais une partie de ces ressources à la gestion de règles édictées par l'administration. En conséquence, la bureaucratie présente au sein des entreprises n'est que le résultat d'interventions étatiques. Ils ne reflètent aucunement des décisions d'entrepreneurs en phase avec le fonctionnement du marché. En effet, ces mesures ne sont pas exigées par le consommateur. Elles constituent ainsi des dépenses improductives qui seraient sanctionnées et corrigées sur un marché libre. Les mécanismes bureaucratiques présents au sein des entreprises ne s'expliquent pas par leur développement ou par l'importance de leur taille, car le calcul économique permet de gérer cette dimension du problème. La bureaucratie résulte donc des règles imposées par l'administration qu'il faut nécessairement appliquer et gérer.

Pour Mises, le système bureaucratique reste néfaste, voire nuisible au bon fonctionnement d'une économie décentralisée. «What is generally castigated as bureaucratic regimentation, pettiness, fearfulness, formalism, and ponderousness is indispensable in an administration that does not seek profit use» (Mises 202 [1932] p. 375). Cette conception tranche avec la version pessimiste de Weber qui y voit un progrès substantiel pour le fonctionnement des sociétés: 
«Precision, speed, unambiguity, knowledge of the files, continuity, discretion, unity, strict subordination, reduction of friction and of material and personal costs - these are raised to the optimum point in the strictly bureaucratic administration, and especially in its monocratic form» (Weber, 1952, p. 216).

\section{Les conséquences politiques et sociales de la bureaucratisation}

Mises caractérise la démocratie par la capacité à destituer politiquement les gouvernants de manière pacifique (Mises 1996 [1962], p. 42). La bureaucratie établir un renversement de perspective concernant la relation entre ceux qui composent le plus grand nombre et ceux qui font partie du plus petit nombre.

En fait, la bureaucratisation des sociétés annonce une nouvelle philosophie. L'individu n'est plus l'élément central dont il faut protéger pour assurer la prospérité. Désormais, l'Etat se situe dans une position surplombante, prenant l'expression d'une forme divine (Mises 2003 (1944) p. 83). L'Etat protégerait contre l'égoïsme de l'individu et constituerait le véhicule privilégie de l'expression du bien commun.

«Aujourd'hui, le culte de l'Etat, philosophie à la mode, a obscurci le problème. Les conflits politiques ne sont plus considérés comme des luttes entre des groupes d'hommes, mais comme une guerre entre deux principes, le bon et le mauvais. Le bon est représenté par le grand dieu Etat, matérialisation de l'idée éternelle de moralité, et le mauvais par "l'âpre individualisme" d'hommes égoïstes. Dans ce dualisme, l'Etat a toujours raison, l'individu toujours tort. L'Etat représente le bien public, la justice, la civilisation et la sagesse supérieure. L'individu est une misérable créature, sotte et méchante» (Ibid. p. 82).

Ce renversement de perspective se traduit également dans les modalités de la relation entre les administrés et les bureaucrates. La position sociale du bureaucrate n'est pas le résultat d'une activité valorisée par le consommateur, mais le produit d'une 
organisation capable de s'arroger des privilèges, qui porte atteinte aux choix du consommateur. Sur un marché libre, le consommateur est souverain pour décider qui peut bénéficier de tels ou tels avantages, de telles ou telles rémunérations. Le comportement d'achat détermine les rémunérations des uns et des autres. Dans un cadre bureaucratique, c'est l'administration elle-même qui décide de sa propre rémunération. Cela est d'autant plus faciliter que le bureaucrate soit également un électeur qui a particulièrement intérêt à se défendre.

«Le bureaucrate n'est pas seulement un fonctionnaire. En régime démocratique, il est en même temps électeur et comme tel participe à la souveraineté de l'Etat qui l'emploie. Sa position est particulière: il est à la fois employeur et salarié. Et son intérêt pécuniaire de salarié l'emporte sur son intérêt d'employeur, car il tire beaucoup plus de la caisse publique qu'il ne contribue à l'accroître.» (Ibid. p. 88).

Non seulement la bureaucratie s'impose à l'individu, avec la complicité du bureaucrate qui se sert en retour de la structure pour bénéficier d'avantages indus, mais la bureaucratisation s'impose dans les esprits par le contrôle de l'éducation qui justifie l'intervention de l'Etat et enseigne les limites du fonctionnement du marché ${ }^{12}$. La bureaucratie s'impose également en développant un appareil de justification intellectuelle promouvant l'intervention de l'Etat. Il existe une véritable dynamique de l'intervention étatique dans les affaires privées, qui trouvent toujours plus de défenseurs à mesure de la croissance de l'Etat et de la multiplication de ses interventions. Il trouve également des partisans dans les bénéficiaires de ses programmes, dans la mesure où l'Etat qui promet l'abondance et l'opulence ne peut retirer à certains au préalable pour donner ensuite à autrui. La situation finale après l'intervention de l'Etat s'avère alors moins satisfaisante que la précédente. L'intervention publique ne pouvant atteindre le but qu'elle s'est fixé, car allant à l'encontre des lois économiques et

12 Cette thèse a été reprise et développée par le Pr. Hoppe dans «Natural Elites, Intellectuals and the State», Ludwig Von Mises Institute, speech delivered at the Supporters Summit in San Francisco, California (1995). 
en menant des politiques qui s'avèrent contradictoires. Toujours plus d'intervention est requise pour faire face au nouvel état d'insatisfaction (Rothbard 1993 [1962], chap. 12).

Progressivement la bureaucratisation transforme la société. Là où le marché avait mis en position de force le consommateur, souverain de sa dépense, elle substitue un ordre politique contraignant décidant pour l'électeur. Dans un système capitaliste, les capitaines d'industrie sont au service des consommateurs (Ibid. p. 97). Désormais le bureaucrate devient le maître qui impose ses propres règles. Les atteintes aux libertés individuelles se multiplient. L'homme constitue alors un moyen pour satisfaire les désirs propres des dirigeants. La menace totalitaire qui accompagne l'omnipotence de l'interventionnisme étatique est dénoncée par Mises par des propos sans ambiguïté.

«Son but n'est pas de rendre les gens heureux, mais de les amener à un état qui le rende heureux, lui dictateur. Il désire les domestiquer, leur donner le rang de bétail.»

"Qui doit être le maître? Tel est le problème. L'homme doit-il être libre de choisir la route qui le mène vers ce qu'il croit le bonheur? Ou le dictateur doit-il utiliser ses semblables comme des pions dans la partie qu'il engage pour devenir lui-même plus heureux?» "Celui qui est incapable de servir ses concitoyens désire les gouverner.» (Mises 2003 (1944) p. 100)

\section{Les conséquences psychologiques}

Le processus de bureaucratisation amène aussi à des conséquences $\mathrm{d}^{\prime}$ ordre psychologique qui se traduisent par l'embrigadement et le contrôle de la jeunesse. Il favorise le conservatisme qui conduit à ôter tout sens critique aux individus.

Mises souligne que l'embrigadement de la jeunesse constitue une des constantes des sociétés fortement bureaucratisées, comme cela a été le cas sous les régimes d'Hitler, de Mussolini et de Staline. Il s'agit de contrôler dès la naissance les citoyens, les obliger à obéir, à étouffer toute contestation. L'adolescent appartient à la mécanique administrative, dont il ne devient qu'un rouage. 
La machine administrative annihile alors chez lui toute velléité révolutionnaire (Ibid. p. 106).

Le système bureaucratique favorise la domination des plus jeunes par les plus anciens. Nous assistons alors à la mie en place d'un système de castes, système que le libéralisme et le capitalisme ont toujours combattu. Ce système de castes conduit à discriminer les individus selon un principe d'ancienneté. La jeunesse doit être soumise, docile et si elle veut réussir, obéissante. Pour réussir, elle doit plaire aux détenteurs de pouvoir, à ceux qui occupent les postes et assurent les responsabilités. Comme l'affirme Mises, «il n'y a pas de place pour les novateurs qui pensent par eux-mêmes» (Ibid. p. 110).

Mises va plus loin dans son analyse et souligne que le processus de bureaucratisation conduit à une crise de civilisation, en bloquant l'évolution et l'amélioration des conditions de vie des individus. Elle stérilise le progrès.

«Nous sommes en présence d'une crise qui n'est pas simplement celle de la jeunesse. C'est une crise du progrès et de la civilisation. L'humanité est condamnée quand la possibilité de refaire la société selon ses propres goûts n'est plus laissée à la jeunesse.» (Ibid. p. 110)

A terme, une société qui fonctionne sur un mode bureaucratique se rigidifie (Ibid. p. 112). La bureaucratie génère un climat de conservatisme social et économique. Elle bloque le processus d'innovation tant sur le plan économique en limitant la prise de risques que sur le plan social en contrôlant les nouvelles idées qui émergeraient des nouvelles générations.

La relation de pouvoir s'en trouve aussi transformée par le passage d'une souveraineté économique permettant le choix de consommation et la satisfaction des besoins les plus urgents à une mise sous tutelle politique avec la mise en œuvre contraignante de réglementations et l'obéissance à des règles définies par les détenteurs de pouvoir. Il existe également un changement de nature. Alors que la relation marchande suppose le respect du contrat passé et de la propriété privée, la relation de pouvoir s'impose par la contrainte. «Le fondement dernier d'un système de bureaucratie généralisée est la violence» (Mises 2003 (1944) p. 114). 
La perte de tout sens critique résulte également de la bureaucratisation des esprits. Le contrôle de l'économie et de la société semble éblouir les intellectuels, fascinés par une telle évolution. Ces derniers démontrent une crédulité et une naïveté confondantes, idéalisant le devenir des sociétés, qu'un dictateur influençable façonnerait conformément à leur propre vision.

«Cette absence d'esprit critique permet de dire aux gens qu'ils seront libres dans un système où tout est réglementé. Ils se figurent qu'un régime où l'ensemble des moyens de production est aux mains de l'Etat et où celui-ci est le seul employeur est le royaume de la liberté. Ils négligent l'éventualité où l'Etat omnipotent de leurs rêves viserait des fins qu'eux-mêmes désapprouveraient totalement. Ils supposent toujours que le dictateur agira exactement dans un sens conforme à leurs désirs» (Ibid. p. 118)

IV

POINTS DE TENSION ET CRITIQUES DE LA THÉORIE AUTRICHIENNE DE LA BUREAUCRATIE

La théorie de la bureaucratie de Mises soulève un certain nombre de remarques et de critiques. Son analyse semble négliger la capacité du bureaucrate à la fois à accroître le budget de son organisation et à bénéficier dans le cadre de son action d'un espace d'incertitude lui permettant de s'abstraire des règles de fonctionnement qui lui sont imposées. Il semble également que la présentation de Mises souffre d'une insuffisance théorique quant aux conditions explicatives de l'extension de la bureaucratie.

\section{Le bureaucrate, un agent capable de s'extraire de la rigidité des règlements?}

Les divergences radicales existant entre l'approche de Mises pouvant être qualifiées d'Autrichienne et celle de Niskanen qui s'inscrit au sein du corpus de l'école des choix publics sont évidentes (Carnis 2000). Néanmoins, l'analyse de Niskanen met 
en avant certaines limites de la théorie de la bureaucratie de Mises.

Pour Niskanen, le bureaucrate recherche à satisfaire ses propres intérêts. En cela, il ne diffère pas du comportement du consommateur ou de tout autre agent qui recherche la maximisation de sa satisfaction. Le bureaucrate n'est donc pas un acteur passif qui obéit à des injonctions transmises par la hiérarchie.

«Most of the literature on bureaucracy, however, has represented the bureaucrat either as an automaton or as maximizing some concept of general welfare, the latter usually considered to be identical with the objectives of the state. For a positive theory of bureaucracy, though, the beginning of wisdom is the recognition that bureaucrats are people who are, at least, not entirely motivated by the general welfare or the interests of the state.» (Niskanen 1994, p. 36)

Le bureaucrate devient ainsi un acteur essentiel au sein des organisations. Il cherche à préserver ses avantages ou à les accroître. Pour cela, les membres de la bureaucratie sont conduits à justifier des augmentations de budget tout en essayant de satisfaire sa tutelle de contrôle (Ibid. p. 42). L'accroissement des budgets s'explique chez Niskanen par une double logique de rationalité et de survie qui est à l'œuvre.

La logique de rationalité suppose que le bureaucrate dispose $d^{\prime}$ 'une fonction de satisfaction, qui intègre des composantes telles que le salaire, des avantages en nature, la réputation, le pouvoir... dont l'importance varie avec la taille du budget géré. Si le bureaucrate désire accroître sa satisfaction, il aura tendance à justifier et à exiger une augmentation des budgets pour permettre de produire en quantité plus importante. Cette production additionnelle nécessitera des embauches supplémentaires assurant des promotions aux titulaires déjà en poste et augmentant les ressources attribuées à l'organisation facilitant son fonctionnement.

«Among the several variables that may enter the bureaucrat's utility function are the following: salary, prerequisites of the office, 
public reputation, power, patronage, output of the bureau, ease of making change, and ease of managing the bureau. All these variables $\ldots$ are a positive monotonic function of the total budget of the bureau during the bureaucrat's tenure in office.» (Ibid. p. 38)

L'augmentation des ressources de l'organisation bureaucratique facilite ainsi l'obtention d'un budget discrétionnaire rendant possible la satisfaction de l'intérêt des bureaucrates (Migué and Bélanger 1974). Cette évolution s'avère d'autant plus facile à justifier pour le bureaucrate du fait de l'impossibilité de spécifier précisément la notion d'intérêt public, puisqu'il n'est pas défini, ni ne fait l'objet d'un consensus. En effet, c'est le bureaucrate luimême qui par son activité décide de fait ce que recouvre cette notion ; il s'agit d'une interprétation toute personnelle qui n'est en rien objective.

Cette conception niskanienne de la bureaucratie pointe le manque de théorisation de la part de Mises concernant le comportement du bureaucrate. Certes ce dernier souligne le caractère intéressé de l'agent administratif, attiré par la sécurité de l'emploi, des pensions flatteuses et des conditions de travail avantageuses associées à sa position. Cependant, l'enchâssement du bureaucrate dans un système de règles, certes existant et contraignant, laisse peu de place pour comprendre son autonomie. Or l'analyse de Niskanen a le mérite de souligner la capacité du bureaucrate à instrumenter sa position pour justifier à la fois des budgets plus importants pour la dépense générale de la production publique et pour obtenir un budget discrétionnaire pour ses fins propres. Il peut ainsi façonner les règles, les moduler et les infléchir. Il existe un espace de liberté que ne conçoit pas Mises, trop influencé sur ce point par la vision de la bureaucratie wéberienne, elle-même critiquée à juste titre (Merton 1939).

La logique de survie souligne les contraintes de l'action du bureaucrate, qui doit composer à l'intérieur de l'organisation avec les autres bureaucrates et à l'extérieur les autres bureaucraties, dont les intérêts diffèrent.

«... a bureaucrat who may not be personally motivated to maximize the budget of his bureau is usually driven by conditions both 
internal and external to the bureau to do just that.» (Niskanen, 1994 p. 39)

Le bureaucrate doit composer avec d'autres bureaucrates au sein des organisations du fait de son appartenance à une hiérarchie. Puisque le nombre de gratifications reste limité, le bureaucrate est incité à œuvrer au développement du budget de son organisation, afin d'accroître le volant discrétionnaire à distribuer. Ce surplus discrétionnaire permet ainsi d'acheter la coopération des bureaucrates subalternes dont le travail est essentiel à l'atteinte des engagements de la bureaucratie (Ibid. p. 40). Le responsable est donc incité à rechercher l'accroissement de la taille de son organisation, afin d'éviter par ailleurs son propre déclassement. Il est d'autant plus incité à le faire que le développement de son organisation constitue un levier essentiel pour accroître son propre prestige et ses prérogatives. Il est également incité à le rechercher pour des motifs externes au fonctionnement de son organisation. Son agence est en concurrence avec d'autres bureaucraties pour l'obtention de budgets. En cela, il recherche l'appui des autorités de tutelle et des politiques ou à le conserver et à l'entretenir. Pour préserver ou obtenir une position privilégiée, le bureaucrate doit proposer de nouveaux projets, qui serviront à alimenter le programme des politiques ou le programme prévisionnel d'actions des autorités.

«They depend on the bureau to seek out and propose new programs and to make a case for larger expenditures in old programs.» (Ibid p. 40)

Les interactions au sein des organisations et entre les organisations conduisent ainsi à l'augmentation de la taille des budgets. La dérive budgétaire s'explique essentiellement par l'hypothèse de la recherche de la satisfaction de l'intérêt personnel du bureaucrate. Même si Mises constate les rivalités et le manque de coordination entre les différentes organisations bureaucratiques (Ibid. p. 93-94), il ne peut percevoir ce jeu d'acteur au sein des organisations. En effet, le strict respect des règles par le bureaucrate est un trait essentiel de sa théorie de la bureaucratie. D'ailleurs, 
de cette caractéristique il dérive une tendance au conservatisme des sociétés fortement bureaucratisée. Cette conclusion s'oppose à la vision développée par Niskanen qui semble suggérer une capacité des bureaucrates à innover, à inventer et à justifier de nouvelles interventions. Par ailleurs, d'autres études sur la bureaucratie soviétique sous Staline, mettent en évidence que même sous des régimes totalitaires, le bureaucrate joue avec des marges de manœuvre quant bien elles restent restreintes.

Cette impossibilité à concevoir un bureaucrate capable $d$ 'initiatives s'explique également par l'adoption d'une vision $d^{\prime}$ 'un bureaucrate mettant en œuvre une expression particulière de l'intérêt public ${ }^{13}$. Les règles sont supposées contrôler toute expression ou recherche de l'intérêt privé. Ikeda propose une typologie des systèmes bureaucratiques qui s'appuie sur une présentation matricielle, croisant les situations en présence d'intérêt personnel ou public, et avec information parfaite ou en ignorance partielle. La théorie de Mises se caractériserait ainsi par la recherche de l'intérêt public et une situation d'ignorance partielle, tandis que l'approche de Niskanen relèverait plus $d^{\prime}$ 'une hypothèse d'intérêt personnel et d'ignorance partielle si l'on tient compte de l'asymétrie d'informations entre agents, voire d'information parfaite si l'on accepte la possibilité d'identifier des fonctions de production. La vision de Mises semble être à la fois victime de l'influence wéberienne et du contexte historique de l'administration de l'Empire prussien, qui s'appuyait sur des bureaucrates dont on louait à la fois le dévouement et la compétence $^{14}$ (Tableau 2).

Il reste que la tendance au conservatisme des sociétés bureaucratisées n'empêche pas Mises de penser la dynamique de l'intervention publique. En effet, cette dynamique s'explique par les contradictions existant entre les différents programmes de

13 Plus précisément, il s'agit des préférences des dirigeants. L'Etat surplombant désormais les égoïsmes individuels, il devient le traducteur de l'intérêt public. Le lecteur peut se référer sur ce point à la partie sur les conséquences politiques, sociales et psychologiques de la bureaucratie.

${ }^{14}$ Le lecteur se reportera avec profit au livre de Marie-Bénédicte Vincent (2006), Serviteurs de l'Etat: les élites administratives en Prusse de 1871 à 1933, Belin. 
TABLEAU 2

MATRICE DES SYSTÈMES BUREAUCRATIQUES

\begin{tabular}{|c|c|c|}
\hline & Intérêt personnel & Intérêt public \\
\hline Connaissance parfaite & Choix publics Chicago & Ecole de l'intérêt public \\
\hline Ignorance partielle & Choix publics Virginie & $\begin{array}{l}\text { Economie politique } \\
\text { autrichienne }\end{array}$ \\
\hline
\end{tabular}

Font: (Ikeda 1997, p. 240).

l'intervention publique d'une part (Mises 2003, p. 94), et de l'incapacité à satisfaire les citoyens avec le nouvel état de la société après la mise en œuvre de l'intervention d'autre part (Ibid. p. 93). Cette insatisfaction appelant de nouvelles interventions. Alors que Mises fonde la dynamique sur les limites du calcul économique dans une économie minée par l'interventionnisme public, Niskanen explique celle-ci par la recherche de l'intérêt personnel du bureaucrate à disposer d'un budget discrétionnaire toujours plus important.

L'approche de Lipsky raisonne en des termes similaires, proches de ceux de Niskanen (Lipsky 1980). Son analyse est essentiellement dirigée vers les comportements des bureaucrates en charge de l'exécution des politiques publiques. Il traite donc des bureaucrates de terrain. Il souligne les conséquences dues à une définition peu ou non précisée des objectifs de politique publique. Cette situation donne une liberté d'interprétation et d'action dans l'exécution de la production publique. Le bureaucrate dispose d'un double degré de liberté. Il est à la fois autonome et dispose d'un pouvoir discrétionnaire (Ibid. chapter 2). Nous retiendrons ici que le bureaucrate peut s'émanciper du contrôle de sa hiérarchie. Il dispose d'une capacité de résistance et peut refuser de coopérer au sein de l'organisation si ses attentes ne sont pas satisfaites (Ibid. p. 16-18). Cette autonomie est âprement défendue par les bureaucrates de terrain face aux tentatives de contrôle éventuelles de leur supérieur. L'exécutant dispose également d'un pouvoir discrétionnaire dans la production de services rendus à la population. Les ressources étant insuffisantes pour satisfaire l'ensemble des demandes, il doit gérer la pénurie à partir de ses 
propres critères et sur lesquels il a la maîtrise. Certes la loi peut déterminer la procédure pour bénéficier de tel service, les conditions d'éligibilité, mais celle-ci ne prévoit pas comment discriminer parmi l'ensemble des bénéficiaires. Ce travail est mis en œuvre par le bureaucrate, qui trouve dans celui-ci une position de force. L'analyse de Lipsky met en lumière les points de tension de la conception de Mises qui évacue cette dimension. Certes Mises souligne qu'une différence entre la gestion marchande et la gestion bureaucratique porte sur le caractère impersonnel des valorisations. Sur un marché, la valorisation porte plus sur des choses que sur les personnes qui produisent les biens (Mises 2003 (1944) p. 43 et 60-61). Les personnes sont jugées sur leur contribution productive, même si des relations plus personnelles peuvent intervenir lors d'une proximité entre le consommateur et le producteur (Ibid. p. 43). La gestion bureaucratique fait quant à elle intervenir des relations de subordination personnelles, qui peuvent se révéler oppressives (Ibid. p. 61). Mises souligne ainsi une certaine souplesse dans le respect des règles. Il s'oppose ainsi à la vision de Weber qui voit dans l'exécution bureaucratique une dépersonnalisation poussée des relations évitant ainsi les problèmes d'intérêts privés.

\section{Théoriser le processus d'expansion bureaucratique}

Le développement de l'interventionnisme et les conditions d'expansion bureaucratique comme modalité d'allocation des ressources s'expliquent chez de Mises par l'incapacité de ce type d'économie à parvenir à un calcul économique élaboré permettant de sélectionner les plans de productions profitables. L'incapacité de l'interventionnisme à satisfaire les désirs qu'il suscite appelle à toujours plus d'intervention.

Cette dynamique peut prendre deux voies divergentes et antagoniques: celle du libre marché et celle de l'économie centralisée qui est condamnée fatalement à l'échec. Or Mises lui-même reconnaît l'existence de plusieurs formes d'interventionnisme et de degré d'intervention (Mises 1998 [1940]; 1996 (1985) [1927]). Même si les différentes expressions de l'interventionnisme peuvent être comprises comme des phases temporaires d'un processus à 
l'œuvre, l'analyse de Mises reste silencieuse pour comprendre les différents états ainsi que les modalités de transition entre ceuxci. Par ailleurs, l'accroissement de l'interventionnisme implique si nous suivons l'argumentation de Mises que l'instrument de calcul économique devient de moins en moins pertinent. Les insatisfactions associées à l'intervention publique deviennent de plus en plus importantes exigeant de nouvelles interventions dégradant d'autant plus la qualité de cet outil. Nous devons en déduire que le rythme de dégradation s'accélère à mesure de l'accroissement des interventions qui elles aussi s'en trouvent accrues pour faire face aux nouveaux problèmes et aux demandes de la population qui surgissent. La vitesse de dégradation de la situation suggère, bien qu'il reste possible de penser des états intermédiaires, un caractère singulièrement instable et peu durable de ces situations. Or la réalité contredit ce résultat, avec des sociétés où la dégradation se fait de manière progressive et à un rythme stabilisé (Ikeda 1997 p. 46), avec des Etats Providences qui perdurent, et des évolutions sociétales qui ne vont pas en sens unique, comme le suggèrent certaines révolutions libérales et managériales.

La non prise en compte de l'autonomie du bureaucrate ne permet pas de rendre compte de la mécanique interne et des arbitrages réalisés au sein de l'organisation publique. Ce point a été mis en évidence précédemment. Par ailleurs, Ikeda souligne également l'importance de la modification des prix relatifs liée à la politique interventionniste, qui pourrait être associée dans une certaine mesure à une altération progressive des préférences (Ibid. p. 133 et 154). Son argumentation consiste à prendre en considération la modification progressive des coûts relatifs de $l^{\prime}$ interventionnisme. Les interventions assurent l'obtention d'avantages pour certains groupes et elles se traduisent également un coût pour d'autres simultanément. Conceptuellement, il existerait alors un équilibre au niveau sociétal plus ou moins stable et favorable à un niveau d'intervention. L'analyse d'Ikeda suggère également une modification des préférences pour l'intervention publique (Ibid. p. 113 et 117). Lorsque celle-ci devient de plus en plus présente, elle peut conduire à une préférence accrue en faveur de l'intervention publique, ce qui faciliterait son 
extension. L'approche d'Ikeda présente l'avantage de comprendre la diversité des interventions et des différences d'intensité. Des sociétés particulières peuvent avoir des préférences différentes à l'égard de l'interventionnisme en y étant plus ou moins favorable. Le rythme de développement de l'interventionnisme s'en trouve également différencié comme d'ailleurs la structure des prix relatifs. Cette théorisation aide à comprendre et à définir des structures de résistance à l'interventionnisme, mais également de rendre possible d'un point de vue conceptuel des retournements. Elle offre également des pistes de compréhension des modalités d'ajustements et de changements d'orientation plus souples sans négliger la situation de rupture (Ibid. p. 123). De fait, Ikeda introduit l'idée de rendements décroissants dans l'usage de l'interventionnisme public, alors que l'analyse de Mises suggérerait des rendements négatifs croissants (la destruction du système se réalise également à un rythme croissant (pertes marginales croissantes)). En cela, cette approche mériterait d'être affinée sur le plan théorique, ce qui rendrait possible l'interprétation des situations intermédiaires et durables des Etats Providences dans le processus de bureaucratisation.

\section{Les limites à la conception d'une bureaucratie minimale}

L'impossibilité de procéder à un calcul économique élaboré constitue l'argument essentiel de Mises pour démontrer le caractère néfaste de la gestion bureaucratique. La bureaucratie, en tant que modalité d'exécution de l'intervention de l'Etat, serait possible seulement pour une sphère d'intervention réduite. Dans ce cas, l'agence gouvernementale peut s'appuyer sur le système de prix relatifs du marché libre pour établir des décisions rationnelles. Pour une situation d'interventionnisme généralisé, l'économie est conduite à un échec inéluctable, «à un appauvrissement général», à «la dissolution de la société et au chaos».

«[Le système bureaucratique] ne réussit qu'à désagréger l'appareil de production et distribution d'un pays. La pauvreté et la misère doivent fatalement en résulter.» (Mises, 2003, (1944) p. 8) 
«Son gaspillage insouciant dissipe la richesse nationale.» (Ibid. p. 7)

Mises limite l'intervention de l'Etat aux fonctions régaliennes de police, de justice et de défense afin d'assurer la coopération sociale (Ibid. p. 23). Cette sphère d'action limitée s'en trouve justifiée à la fois pour des motifs de praticabilité de l'intervention elle-même et la nécessité de disposer des informations requises pour procéder aux décisions idoines. Elle s'explique également par les limites du marché à prendre en charge de telles productions. En effet, la production de ces biens et services par l'Etat résulterait d'une caractéristique d'incommensurabilité de l'extrant et ne saurait faire l'objet d'un calcul économique. Il s'agit de protéger la liberté et les droits des individus (Ibid. p. 51).

Cette justification enclenche donc le processus d'une première intervention de l'Etat qui nécessite le prélèvement et l'utilisation de ressources, pour assurer leur financement, ce qui accroît d'un degré supplémentaire l'intervention publique initiale. La conséquence de la justification de domaines réservés de l'Etat conduit à autoriser d'autres immixtions dans des secteurs connexes qui alimenteront l'intervention au sein d'autres secteurs. La dynamique interventionniste serait enclenchée. En effet, ces interventions, si nous suivons le schéma de Mises, doivent conduire à des distorsions sur le marché qui susciteront des appels à de nouvelles interventions. En somme, l'argumentation de Mises en faveur d'une bureaucratie minimale est porteuse de dangers réels en permettant d'initier un mécanisme dont luimême dénonce les conséquences.

L'argument d'incommensurabilité n'est pas satisfaisant pour justifier l'intervention publique dans ces domaines, dans la mesure où le marché intervient dans de nombreux secteurs où les individus doivent établir des arbitrages impliquant une telle dimension ${ }^{15}$ : éducation des enfants, système de sécurité, hygiène alimentaire... L'argument lui-même peut ouvrir la porte à de nouvelles formes d'intervention en étendant la notion de sécurité aux domaines de la santé (contrôle des ventes d'alcool, de tabac,

\footnotetext{
15 La production échapperait à un arbitrage en monnaie.
} 
politiques de santé...), de conduites à risque (contrôle des vitesses de circulation, risques financiers et endettement...), à celles de justice et de justice sociale (favoriser la redistribution des revenus pour éviter qu'elle ne s'effectue par la violence), favoriser la politique de stabilité économique pour protéger les individus d'effets éventuels liés aux changements conjoncturels et structurels... Par ailleurs, l'argument qui postule l'incapacité du secteur privé à intervenir dans tels secteurs est contredit par un certain nombre de théories (Hoppe 1999) (Rothbard 1996 (1973)) (Tannehill 1984 (1970) et de faits sociaux (importance du secteur privé dans le secteur de la sécurité, firmes privées de production d'armement, système privé d'éducation, de santé et d'assurance couvrant de nombreux risques). Le rejet de la conception anarchiste ou libertarienne par Mises ne nous semble pas reposer sur une argumentation logique et ouvre la porte à des contradictions au sein même de l'approche (Mises 1986, p. 37).

Mises précise que la gestion bureaucratique échappe au contrôle du calcul économique (Mises, 2003 (1944), p. 54). La formulation s'avère relativement ambiguë dans la mesure où il n'est pas précisé si cela est la conséquence ou la cause de l'intervention. L'intervention s'explique-t-elle par ce que le calcul économique ne peut faire l'objet d'une application dans ce cas précis? La gestion bureaucratique conduit-elle par sa nature à exclure toute possibilité de calcul dans le domaine d'intervention précis? En somme, la production échappe-t-elle à une évaluation par le marché parce qu'elle ne peut faire l'objet de transactions marchandes ou parce qu'elle s'y soustrait?

«Les objectifs des administrations publiques ne sont susceptibles d'évaluation en termes monétaires et ne se soumettent pas au contrôle des méthodes comptables.» (Mises 2003 (1944) p. 52)

Il semblerait que Mises partage sur ce point une position ambivalente. L'incapacité à évaluer en termes monétaires une production serait l'explication de l'intervention pour ce qui concerne des domaines circonscrits assurant la coopération sociale et d'une conséquence pour toute intervention qui irait 
au-delà de ce périmètre défini ${ }^{16}$. Cette explication duale de l'intervention publique prête le flanc non seulement à de sévères critiques comme celles mentionnées dans le point précèdent, mais aussi comment devient-il désormais possible de justifier cette différence? Mises n'avance aucune précision sur ce point, ce qui en affaiblit l'argumentation. L'engagement de gardes du corps assurant la sécurité physique des personnes ne peut-il pas faire l'objet de transactions sur le marché? La gestion de la santé par le secteur privé, de l'éducation des enfants par les parents ne comprend-il pas des dimensions incommensurables qui pourraient justifier l'intervention de l'Etat? Mises laisse également la porte ouverte à d'autres interprétations de l'intervention publique. Certains pourraient arguer que l'intervention publique est requise dans tel domaine du fait de l'impossibilité d'utiliser le calcul économique au motif de considérations morales. Dans ce cas, le raisonnement en termes de rentabilité des plans mis en œuvre ne se poserait pas, puisque par nature il ne pourrait s'y appliquer. En conséquence, la supériorité de la procédure de marché sur le mode de gestion bureaucratique ne pourrait être avancée, puisque nous aurions renoncé alors à ce critère. La démonstration empirique de l'échec d'un système par rapport à un autre ne pourrait plus être tranchée, comme pour délibérer sur les échecs de l'économie mixte. La justification ultime reposerait alors sur un jugement de valeur émanant de la part du dirigeant et des bureaucrates. L'argumentation de Mises nécessite ainsi d'être raffinée notamment en précisant le fonctionnement de l'organisation bureaucratique et en abandonnant la défense d'un domaine réservé de l'intervention publique ou en en trouvant une justification plus cohérente avec l'ensemble théorique proposé.

16 Or comme nous l'avons précédemment remarqué, la première intervention risque fort de constituer un cheval de Troie de l'interventionnisme dans le fonctionnement du libre marché. Cette perspective suggère également l'existence d'une imbrication inévitable. 


\section{$\mathrm{V}$ \\ CONCLUSION}

L'analyse de la bureaucratie proposée par Mises s'inscrit dans une réflexion générale sur l'interventionnisme public et ses conséquences sur le fonctionnement de l'économie de marché. Mises associe dans son approche la théorie de l'entrepreneur et le problème de l'économie de marché. Les deux s'articulent pour produire les biens demandés tout en réalisant la production au moindre coût. L'économie de marché sert ainsi de référentiel pour comprendre les conséquences de la gestion bureaucratique, dont les caractéristiques génériques présentent une certaine proximité avec la conception de Weber.

La bureaucratie se conçoit chez Mises comme une technique de mise en œuvre de l'intervention publique, qui conduit inévitablement au délabrement économique et au chaos social en détruisant la coopération entre les personnes. Le risque politique n'est pas négligeable puisqu'il peut dériver rapidement vers un système totalitaire. Mises a clairement identifié les raisons de cette incapacité de la bureaucratie à opérer efficacement. L'absence du mécanisme de profit et le non-sens du calcul public, lorsqu'il est mis en oeuvre ne permet pas de satisfaire les consommateurs. Une destruction de richesses doit s'en suivre. Les conséquences sociales et psychologiques ne sont pas moins importantes, puisqu'il s'agit ni plus ni moins de la mise sous tutelle progressive d'une société qui s'en trouve dès lors condamnée à la sclérose, à l'immobilisme, annihilant les ressorts que sont la jeunesse, l'innovation, la constitution d'un capital et qui permettent de bâtir un avenir pour la société. La fin ultime conduit alors à un inéluctable déclin et à la destruction de la société elle-même.

Il reste que cette approche présente certaines apories qui exigent de nouveaux développements. Cet effort pourrait prendre la forme d'une nouvelle élaboration d'une théorie autrichienne de la bureaucratie, prenant racine dans les travaux précurseurs de Mises et de Rothbard, tout en se démarquant de la conception plus en vogue développée par l'école des choix publics. 


\section{BIBLIOGRAPHIE}

Alchian A. and H. Demsetz 1973, «The Property Right Paradigm», Journal of Economic History, (43): 16-27.

ANDERSON WiLliam P. 2004, «Mises versus Weber on Bureaucracy and Sociological Method», Journal of Libertarian Studies, (18)1: 1-29, winter.

Archibald G.C. 1971. The Theory of the Firm, Penguin Modern Economics.

Barzel Yoram. 2003. «Property Rights in the Firm» in Terry L. Anderson and Fred S. McChesnay (eds.), Property Rights, Cooperation, Conflict and Law, Princeton University Press, (43-57).

Breton Albert and Ronald Wintrobe. 1982. The Logic of Bureaucratic Conduct, An Economic Analysis of Competition, Exchange, and Efficiency in Private and Public Organizations, Cambridge University Press.

- 1975. «The Equilibrium Size of a Budget-Maximizing Bureau: A note on Niskanen's Theory of Bureaucracy», Journal of Political Economy, (83): pp. 195-203.

CARnis LAURENT. 2000. «La théorie de la bureaucratie: apports de

Mises et essai comparatiste» in Association des Historiens de Tradition Autrichienne, Autour des développements récents de l'économie autrichienne, Les cahiers du Ceras, octobre n.. 39, pp. 8-30.

Coase Ronald H. 2000. Le coût du droit, Collection Droit, Economie, Société, Presses Universitaires de France.

- 1960. «The Problem of Social Cost», The Journal of Law and Economics, Vol. III, October, pp. 1-44.

- 1937. "The Nature of the Firm», Economica, (4), November. Downs Anthony. 1994 [1966]. Inside Bureaucracy, Waveland Press. GrefFe Xavier. 1994. Economie des politiques publiques, Dalloz, Sciences économiques.

GrefFe Xavier. 1981. Analyse économique de la bureaucratie, Economica. HAYEK FRIEDRICH (VON). 1993 [1944]. La route de la servitude, Presses Universitaires de France, Collection Quadrige.

Hoppe Hans-Hermann. 1999. The Private Production of Defense, Ludwig von Mises Institute, Auburn, Alabama. 
HÜLSMANN JöRG GuIDO. 1998. «Toward a General Theory of Error Cycles», The Quarterly Journal of Austrian Economics, 1(4): 1-23 (winter).

IKEDA SANFORD. 1994. "Interventionism» in Peter J. Boettke (ed.) The Elgar Companion to Austrian Economics, chapter 49, p. 345-351.

- 1997. Dynamics of the Mixed Economy, Toward a Theory of Interventionism, Foundations of the Market Economy, Routledge.

IoANNides StaVros. 1999. «Towards An Austrian Perspective on the Firm», Review of Austrian Economics, (11): 77-97.

Lipsky Michael. 1980. Street Level Bureaucracy, The Dilemmas of the Individual in Public Services, Russell Sage Foundation.

Migué JeAn-Luc et Gérard Bélanger. 1974. «Towards a General Theory of Managerial Discretion», Public Choice (17).

Mises Ludwig (von). 2003 [1944]. La bureaucratie, Editions de l'Institut Charles Coquelin.

- 2002 [1932]. Selected Writings of Ludwig von Mises, Economic calculation under Commercial Management and Bureaucratic Administration, chapter 43, pp. 372-379 Volume 2, edited by Richard M. Ebeling, Liberty Fund.

- 1998 [1940]. Interventionism, An Economic Analysis, Foundation for Economic Education.

- 1996 (1985) (1962) [1927]. Liberalism, The Classical Tradition, Foundation for Economic Education.

- 1990. Economic Calculation in the Socialist Commonwealth, Ludwig von Mises Institute.

- 1983 (1969) [1944]. Bureaucracy, Libertarian Press.

MERTON RoBert. 1939-1940. «Bureaucratic Structure and Personality», Social Forces, Vol. 18: 560-568.

Niskanen William, A. 1994. Bureaucracy and Public Economics, The Locke Institute.

- 1975. «Bureaucrats and Politicians», Journal of Law and Economics, (18): 617-643.

- 1968. «The Peculiar Economics of Bureaucracy», American Economic Review, LVIII(3): 293-305.

Peltzman SAm. 1980. «The Growth of Government», Journal of Law and Economics, (23)2. 
Pierson N. G., Ludwig von Mises, Georg Halm et Enrico Barone. 1939. L'économie dirigée en régime collectiviste, Etudes critiques sur les possibilités du socialisme, Librairie de Mèdicis.

Reisman George. 1998. Capitalism, A Complete and Integrated Understanding of the Nature and Value of Human Economic Life, Jameson Books.

Rothbard Murray N. 1993 [1962]. Man, Economy, and State, A Treatise on Economic Principles, Ludwig Von Mises Institute.

- 1996 [1973]. For a New Liberty, The Libertarian Manifesto, Fox and Wilkes Revised Edition.

SAUTET FrÉDÉRIC E. 2000. An Entrepreneurial Theory of the Firm, Routledge, Foundations of the Market Economy.

TANNEHILl Linda AND MoRRIs. 1993 [1970]. The Market for Liberty, Fox and Wilkes.

Tullock Gordon. 1965. The Politics of Bureaucracy, Public Affairs Press.

Weber Max. 1952. From Max Weber: Essays in Sociology, Routledge and Kegan Paul Ltd. 